\title{
Growth Differentiation Factor 15 Predicts Chronic Liver Disease Severity
}

\author{
Eaum Seok Lee ${ }^{1}$, Seok Hyun Kim ${ }^{1}$, Hyun Jin Kim ${ }^{1}$, Kyung Hee Kim² ${ }^{2}$ Byung Seok Lee ${ }^{1}$, and Bon Jeong Ku ${ }^{1}$ \\ Departments of ${ }^{1}$ Internal Medicine and ${ }^{2}$ Pathology, Chungnam National University School of Medicine, Daejeon, Korea
}

Background/Aims: Growth differentiation factor 15 (GDF15) belongs to the transforming growth factor- $\beta$ superfamily. GDF-15 is emerging as a biomarker for several diseases. The aim of this study was to determine the clinical performances of GDF-15 for the prediction of liver fibrosis and severity in chronic liver disease. Methods: The serum GDF-15 levels were examined via enzyme immunoassay in 145 patients with chronic liver disease and 101 healthy individuals. The patients with chronic liver disease consisted of 54 patients with chronic hepatitis, 44 patients with compensated liver cirrhosis, and 47 patients with decompensated liver cirrhosis. Results: Of the patients with chronic liver diseases, the decompensated liver cirrhosis patients had an increased serum GDF-15 (3,483 ng/L) level compared with the patients with compensated liver cirrhosis $(1,861 \mathrm{ng} / \mathrm{L})$ and chronic hepatitis $(1,232 \mathrm{ng} / \mathrm{L})$. The overall diagnostic accuracies of GDF-15, as determined by the area under the receiver operating characteristic curves, were as follows: chronic hepatitis=0.656 (>574 ng/L, sensitivity, 53.7\%; specificity, 79.2\%), compensated liver cirrhosis=0.886 (>760 ng/L, sensitivity, 75.6\%; specificity, 92.1\%), and decompensated liver cirrhosis=0.984 (>869 ng/L, sensitivity, 97.9\%; specificity, 94.1\%). Conclusions: This investigation represents the first study to demonstrate the availability of GDF-15 in chronic liver disease. GDF-15 comprised a useful biomarker for the prediction of liver fibrosis and severity in chronic liver disease. (Gut Liver 2017;11:276-282)

Key Words: Chronic liver disease; Decompensated liver cirrhosis; Fibrosis; Growth differentiation factor 15; Biomarkers

\section{INTRODUCTION}

Chronic liver disease has high global mortality rates. However, clinical outcomes are diverse due to liver remnant synthesis, causes, and the occurrence of hepatocellular carcinoma, making prognosis difficult to predict. In patients with chronic liver disease, it is critical to have a clear understanding of hepatic fibrosis and its severity because it could help clinicians predict patient prognosis and choose a liver transplant date. Over the past 20 years, many prognosis prediction models have been suggested, but each had various limitations, creating the need for a simpler and more accurate biomarker. From the clinical point of view, distinguishing between compensated and decompensated cirrhosis when predicting patient prognosis is important. However, current models including Child-Turcotte-Pugh (CTP) and Model for End-stage Liver Disease (MELD) scores cannot distinguish between compensated and decompensated cirrhosis. ${ }^{1}$

Growth differentiation factor 15 (GDF-15) is a transforming growth factor $\beta$ (TGF- $\beta$ ) protein involved in infection, fibrosis, and apoptosis pathways in the presence of tissue damage or disease. GDF-15 is also known as TGF-PL (placental), macrophage inhibitory cytokine-1, prostate derived factor, placental bone morphogenetic protein, and placental growth factor- $\beta$, and its mRNA is known to be distributed particularly in the liver but also in the kidneys, heart, and lungs. In the presence of hypoxia, anoxia, inflammation, short-wave length exposure, and tissue injuries, the GDF-15 gene is expressed by activated macrophages and GDF-15 protein expression increases. ${ }^{2}$ The mechanisms behind this are not clearly known, but they are thought to be related to inflammation by CCR2-mediated chemotaxis and known to be involved with connective tissue fibrosis. ${ }^{3}$

This study aimed to investigate the effectiveness of GDF-15

\footnotetext{
Correspondence to: Byung Seok Lee ${ }^{\mathrm{a}}$ and Bon Jeong $\mathrm{Ku}^{\mathrm{b}}$

${ }^{a}$ Division of Gastroenterology \& Hepatology, Department of Internal Medicine, Chungnam National University School of Medicine, 266 Munhwaro, Jung-gu, Daejeon 35015, Korea

Tel: +82-10-9344-9763, Fax: +82-42-255-4553, E-mail: gieoo1@cnuh.co.kr

${ }^{\mathrm{b}}$ Division of Endocrinology \& Metabolism, Department of Internal Medicine, Chungnam National University School of Medicine, 266 Munhwa-ro, Jung-gu, Daejeon 35015, Korea

Tel: +82-10-8810-6031, Fax: +82-42-280-7995, E-mail: bonjeong@cnu.ac.kr

Received on January 25, 2016. Revised on May 7, 2016. Accepted on May 17, 2016. Published online 0ctober 13,2016

pISSN 1976-2283 eISSN 2005-1212 https://doi.org/10.5009/gnl16049

Byung Seok Lee and Bon Jeong Ku equally supervised this work.

@ This is an Open Access article distributed under the terms of the Creative Commons Attribution Non-Commercial License (http://creativecommons.org/licenses/by-nc/4.0) which permits unrestricted non-commercial use, distribution, and reproduction in any medium, provided the original work is properly cited.
} 
for predicting liver disease severity and fibrosis as well as the applicability of biomarkers in patients with chronic liver diseases, which are primarily caused by inflammation and fibrosis.

\section{MATERIALS AND METHODS}

\section{Patients}

A total of 101 patients admitted for medical check-ups between August 2013 and December 2013 were included in the control group, while 145 patients with chronic liver diseases were included in the patient group and identified as having chronic hepatitis, compensated cirrhosis, or decompensated cirrhosis.

Among the patients with chronic liver diseases, patients with hepatocellular carcinoma were excluded from the study. Patients on anti-inflammatory drugs such as aspirin and steroids, diagnosed with other cancers, or with autoimmune diseases such as gout and rheumatic arthritis were also excluded from this study because these factors could affect GDF-15 concentrations. In addition, considering the traditional technology that could use GDF-15 as the biomarker for infections, patients with damaged liver cells from past infections in the past were tested, and the result showed that the GDF-15 blood concentrations were uniformly increased. Hence, patients with toxic or acute hepatitis were also excluded from this study.

Decompensated cirrhosis was defined as the patient having complications such as variceal bleeding, ascites, encephalopathy, and jaundice. ${ }^{4}$ Compensated cirrhosis was identified when patients did not display any of the aforementioned complications.

\section{Methods}

Once informed consent is obtained, demographical characteristics, clinical characteristics, and laboratory results of each group were investigated and analyzed and the patients were followed on an outpatient basis. Blood was collected from patients who agreed to genetic testing. The study was reviewed and approved by Chungnam National University School of Medicine, Institutional Review Board.

The blood concentration of GDF-15 measured by enzymelinked immunosorbent assay (ELISA) using a human GDF-15 ELISA kit (R\&D Systems Inc., Minneapolis, MN, USA). Body mass index (BMI), aspartate aminotransferase (AST), alanine aminotransferase (ALT), cirrhosis discriminant score (CDS), Child-Pugh score, and MELD score were measured to compare them with other indexes. BMI was calculated using the formula (weight $[\mathrm{kg}] /$ height $\left[\mathrm{m}^{2}\right]$ ).

The blood specimens were centrifuged, and AST and ALT activity and blood concentration analyses were performed of the supernatant. The blood analysis kit was then used according to the manufacturer's instructions. CDS measured the number of platelets in the blood specimen and the prothrombin time. The obtained score was added accordingly to the score system and then classified.

The Child-Pugh score was based on the sum of the scores from following systems: (1) serum bilirubin $<2 \mathrm{mg} / \mathrm{dL}$ (score 1), 2 to $3 \mathrm{mg} / \mathrm{dL}$ (score 2), $>3 \mathrm{mg} / \mathrm{dL}$ (score 3); (2) serum albumin $>3.5 \mathrm{~g} / \mathrm{dL}$ (score 1), 2.8 to $3.5 \mathrm{~g} / \mathrm{dL}$ (score 2), <2.8 g/dL (score 3); (3) prothrombin time international normalized ratio (PT INR) $<1.70$ (score 1), 1.71 to 2.20 (score 2), >2.20 (score 3); (4) no ascities (score 1), adjustable ascites (score 2), nonadjustable ascites (score 3); (5) no hepatic encephalopathy (score 1), easily treated hepatic encephalopathy (score 2), noneasily treated hepatic encephalopathy (score 3).

MELD score was calculated by the equation of $9.6 \times \ln$ (creatinine $\mathrm{mg} / \mathrm{dL})+3.8 \times \ln ($ bilirubin $\mathrm{mg} / \mathrm{dL})+11.2 \times \ln (\mathrm{INR})+6.4$.

\section{Statistical analysis}

The resulting values are expressed as mean \pm standard deviation and the statistical analyses were performed using SPSS for Windows version 21 (IBM Corp., Armonk, NY, USA). The pvalues $<0.05$ were considered statistically significant.

Patient gender, disease causes, and clinical information were compared using Fisher exact test, while the $\times 2$ chi square test was used for categorical data. The correlation among GDF-15, CDS, MELD score, and CTP score was replaced by the log value and analyzed using Spearman correlation coefficients.

Determinates of GDF-15 levels were tested using a univariate binary logistic regression analysis. All variables, which were tested significant in the univariate model, were inserted in a multivariate binary logistic regression model with inclusion method. Results of binary logistic regression analysis were presented as odds ratio and $95 \%$ confidence interval (CI).

To confirm the effectiveness of GDF-15 in diagnosis, the receiver operating characteristic (ROC) curve was used to determine the area under the ROC curve. The cutoff value was set up to find the sensitivity and specificity as well as the predictability for positives and negatives.

\section{RESULTS}

\section{Clinical characteristics of the patient group}

A total of 246 blood samples collected for this study from 101 healthy adults and 145 patients with chronic liver disease were used, and the participants' basic characteristics are shown in Table 1.

The control group consisted of 55 men and 46 women $(n=101)$. The ratios of men to women in the chronic hepatitis, compensated cirrhosis, and decompensated cirrhosis groups were 28/26, $26 / 18$, and 18/29, respectively, and no significant difference was seen among groups ( $\mathrm{p}=0.741)$.

The average age of the control group was $46 \pm 9$ years, while those of the chronic hepatitis, compensated cirrhosis, and decompensated cirrhosis groups were $45 \pm 13,59 \pm 10$, and $56 \pm 12$ 
Table 1. Characteristics of Study Participants $(n=246)$

\begin{tabular}{|c|c|c|c|c|c|}
\hline & $\begin{array}{l}\text { Healthy person } \\
\qquad(n=101)\end{array}$ & $\begin{array}{l}\text { Chronic hepatitis } \\
\qquad(\mathrm{n}=54)\end{array}$ & $\begin{array}{l}\text { Compensated LC } \\
\qquad(\mathrm{n}=44)\end{array}$ & $\begin{array}{l}\text { Decompensated LC } \\
\qquad(\mathrm{n}=47)\end{array}$ & p-value \\
\hline $\operatorname{Sex}(M / F)$ & $55 / 46$ & $28 / 26$ & $26 / 18$ & $18 / 29$ & 0.741 \\
\hline Age, yr & $46 \pm 9$ & $45 \pm 13$ & $59 \pm 10$ & $56 \pm 12$ & $<0.01$ \\
\hline \multicolumn{6}{|l|}{ Etiology } \\
\hline $\mathrm{HBV}$ & - & $39(72.2)$ & $22(50)$ & $8(17.0)$ & - \\
\hline $\mathrm{HCV}$ & - & $12(22.2)$ & 7 (15.9) & $8(17.0)$ & - \\
\hline Alcohol & - & $3(5.6)$ & 7 (15.9) & 27 (57.4) & - \\
\hline Others & - & 0 & $8(18.2)$ & $4(8.6)$ & - \\
\hline BMI & $24.2 \pm 2.94$ & $24.1 \pm 3.7$ & $22.8 \pm 5.9$ & $24.1 \pm 3.6$ & 0.382 \\
\hline AST & 22 & 44 & 50 & 41 & 0.002 \\
\hline ALT & 24 & 56 & 42 & 22 & 0.046 \\
\hline CDS & - & - & $6.7 \pm 1.6$ & $8.7 \pm 1.7$ & $<0.01$ \\
\hline Child-Pugh score & - & - & $5.3 \pm 0.7$ & $8.2 \pm 1.9$ & $<0.01$ \\
\hline MELD score & - & - & $8.7 \pm 2.7$ & $13.8 \pm 4.7$ & $<0.01$ \\
\hline GDF-15, ng/L & 490 & 1,232 & 1,861 & 3,483 & $<0.01$ \\
\hline
\end{tabular}

Data are presented as number (\%) or mean \pm SD.

LC, liver cirrhosis; M, male; F, female; HBV, hepatitis B virus; HCV, hepatitis C virus; BMI, body mass index; AST, aspartate aminotransferase; ALT, alanine aminotransferase; CDS, cirrhosis discriminant score; MELD score, Model for End-stage Liver Disease; GDF-15, growth differentiating factor 15.

Table 2. Multivariate Analysis of Blood GDF-15 Concentration Using a Multivariate Logistic Regression Test

\begin{tabular}{lrccc}
\hline & No. & OR & $95 \%$ CI & p-value \\
\hline Chronic liver disease $^{*}$ & 145 & 1.002 & $1.001-1.002$ & 0.000 \\
Chronic hepatitis & 54 & 1.001 & $1.000-1.002$ & 0.011 \\
Compensated LC & 44 & 1.001 & $1.000-1.002$ & 0.009 \\
Decompensated LC & 47 & 1.002 & $1.001-1.003$ & 0.002 \\
\hline
\end{tabular}

Adjusted for significant variables, including age, aspartate aminotransferase, alanine aminotransferase, Child-Pugh score, and Model for End-stage Liver Disease (MELS) score.

GDF-15, growth differentiating factor 15; OR, odds ratio; CI, confidence interval; LC, liver cirrhosis.

*Included chronic hepatitis, compensated LC, and decompensated LC.

years, respectively, showing that those in the control group and chronic hepatitis group were significantly younger $(\mathrm{p}<0.01)$.

Causes of chronic liver disease in the 54 patients with chronic hepatitis consisted of hepatitis B virus ( $n=39)$, hepatitis C virus $(\mathrm{n}=12)$, and alcohol intake $(\mathrm{n}=3)$, while causes of compensated cirrhosis included hepatitis B virus ( $\mathrm{n}=22)$, hepatitis $\mathrm{C}$ virus $(\mathrm{n}=7)$, and alcohol $(\mathrm{n}=7)$, and other autoimmune diseases or unknown causes $(\mathrm{n}=8)$ and causes of decompensated cirrhosis included hepatitis B virus $(n=8)$, hepatitis C virus $(n=8)$, alcohol $(n=27)$, and other $(\mathrm{n}=4)$, respectively.

The average BMI of the control and chronic liver diseases groups were $24.2 \pm 2.94,24.1 \pm 3.7,22.8 \pm 5.9$, and $24.1 \pm 3.6$, respectively; there were no statistically significant differences among groups ( $p=0.382)$. The average AST level was higher in the liver disease group than in the control group, but there was no major difference seen among the liver disease subgroups. The average ALT level was higher in the chronic hepatitis and compensated cirrhosis groups than in the control and decompensated cirrhosis groups.

The CDS, CTP score, and MELD score, which can predict liver fibrosis and severity in patients with cirrhosis, were $6.7 \pm 1.6$ versus $8.7 \pm 1.7,5.3 \pm 0.7$ versus $8.2 \pm 1.9$, and $8.7 \pm 2.7$ versus $13.8 \pm 4.7$, respectively, significantly higher in the patients with decompensated cirrhosis $(\mathrm{p}<0.01)$.

In a multivariate analysis, GDF-15 levels were independent predictors of chronic liver diseases (Table 2).

\section{Blood GDF-15 concentration in patients with chronic liver diseases}

When the blood GDF-15 concentration was measured within the $95 \%$ CI of each patient group, the average value in the control group was $490 \mathrm{ng} / \mathrm{L}$, but that value rapidly increased in the chronic liver disease patients to $>1,000 \mathrm{ng} / \mathrm{L}$. In particular, GDF15 concentration in the decompensated cirrhosis group was $3,483 \mathrm{ng} / \mathrm{L}$, which was clearly higher than that in the chronic hepatitis patients at 1,232 ng/L and that in the compensated cirrhosis patients at $1,861 \mathrm{ng} / \mathrm{L}$, indicating a statistically significant difference $(\mathrm{p}<0.01)$.

\section{Correlation between serum marker assessment (MELD score, CTP score, and CDS) in liver fibrosis and severity prediction and GDF-15}

When the significant serum markers from the analysis of correlation between the factors that could predict fibrosis and 

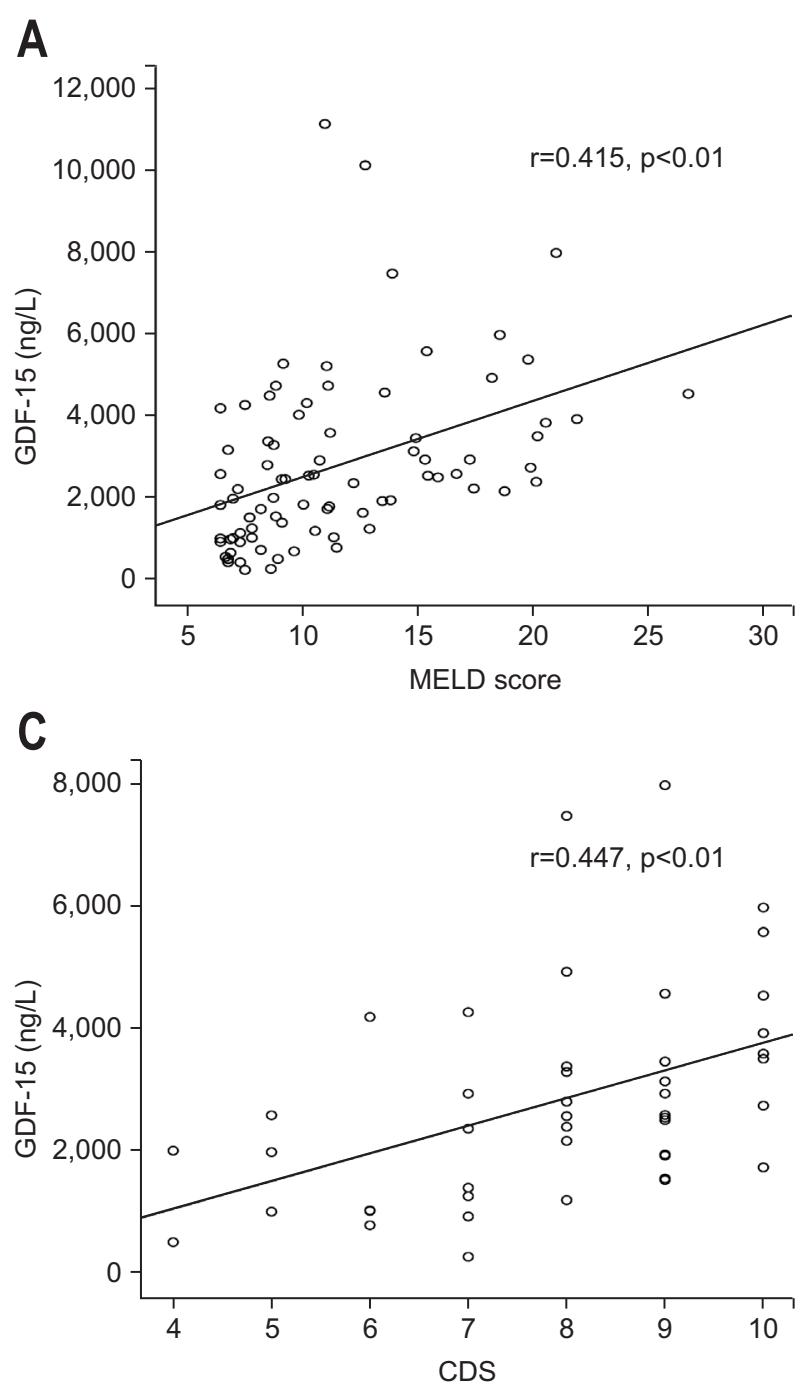

liver disease severity and GDF-15 were ranked from the highest bivariate Spearman rank correlation coefficient to the lowest, the results were as follows: Child-Pugh score ( $r=0.474)$, CDS $(\mathrm{r}=0.447)$, and MELD score $(\mathrm{r}=0.415)(\mathrm{p}<0.01)$ (Fig. 1). Furthermore, Child-Pugh score and MELD score showed the highest positive correlation of 0.873 .

\section{AUC value of GDF-15 in predicting chronic liver disease severity}

The AUC value of GDF-15 in the ROC curves for chronic hepatitis, compensated liver cirrhosis, and decompensated liver cirrhosis patients were $0.656,0.886$, and 0.984 , respectively (Fig. 2).

\section{Sensitivity, specificity, and predictability for positives and negatives of GDF-15 in predicting severity in chronic liver diseases}

On the ROC curve, the value with highest specificity and rapid decrease in sensitivity was set as the optimal cutoff value. When the optimal cutoff value was set as $574 \mathrm{ng} / \mathrm{L}$ for patients with chronic hepatitis, the predictability of GDF-15 had a sensitiv-

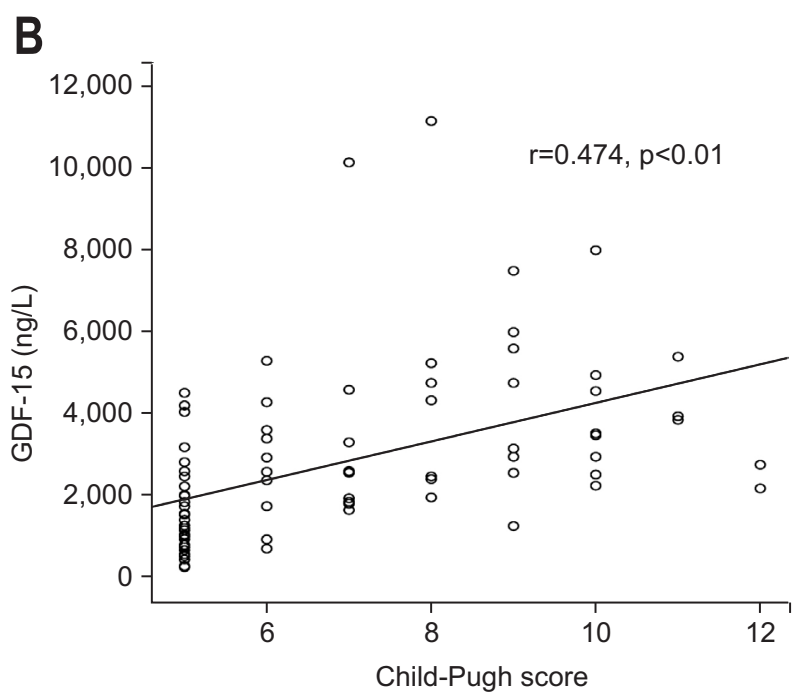

Fig. 1. (A) Correlation between the Model for End-stage Liver Disease (MELD) score and growth differentiation factor 15 (GDF-15); (B) Correlation between the Child-Pugh score and GDF-15; (C) Correlation between the cirrhosis discriminant score (CDS) and GDF-15. When the significant serum markers from the analysis of the correlations between the factors that predicted fibrosis and liver disease severity and GDF-15 were ranked from the highest bivariate Spearman rank correlation coefficient to the lowest, the results were as follows: Child-Pugh score ( $r=0.474)$, CDS $(r=0.447)$, and MELD score $(r=0.415)$ $(\mathrm{p}<0.01)$.

ity of 53.7\%, specificity of 79.2\%, predictability for positives of $58.0 \%$, and predictability for negatives of 76.2\%.

When the optimal cutoff value was set at $760 \mathrm{ng} / \mathrm{L}$ for patients with compensated liver cirrhosis, the predictability of GDF-15 had a sensitivity of 75.6\%, specificity of $92.1 \%$, predictability for positives of $81.0 \%$, and predictability for negatives of $89.4 \%$. When the optimal cutoff value was set at $869 \mathrm{ng} / \mathrm{L}$ for patients with decompensated liver cirrhosis, the predictability of GDF-15 had a sensitivity of 97.9\%, specificity of 94.1\%, predictability for positives of $88.5 \%$, and predictability of negatives of 99.0\% (Table 3).

\section{DISCUSSION}

Some new facts were discovered through this study. First, patients with chronic liver diseases had higher GDF-15 concentrations than patients in the control group, and the patients with more severe liver diseases showed proportionally higher GDF-15 values. To date, studies have reported that a high serum GDF-15 value was related to lymph node metastasis and a low survival 

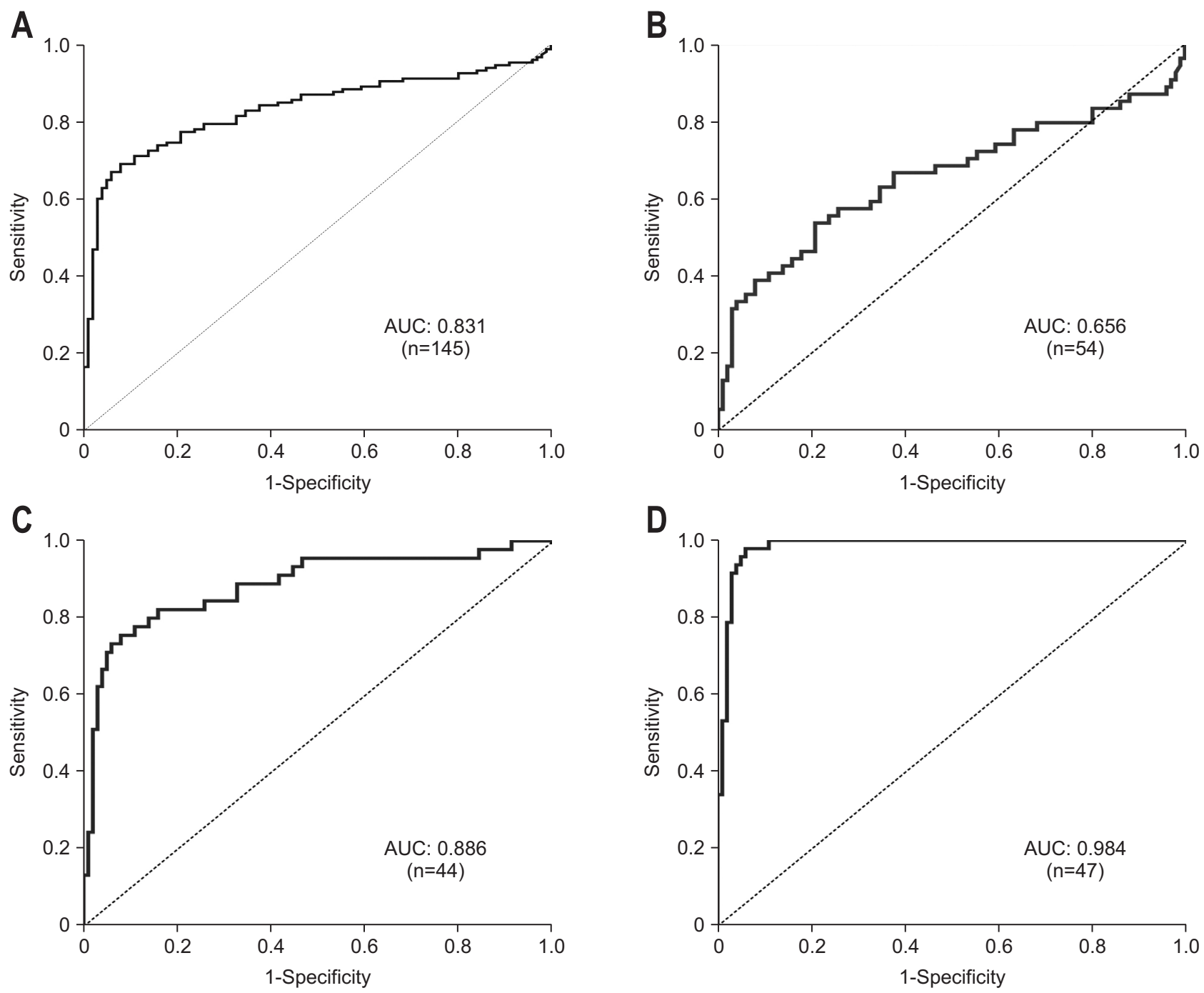

Fig. 2. Receiver operating characteristic (ROC) curve of the growth differentiation factor 15 (GDF-15) value in patients with chronic liver diseases (A), chronic hepatitis (B), compensated liver cirrhosis (C), and decompensated liver cirrhosis (D). The area under the curve (AUC) value of GDF-15 in the ROC curves for chronic liver disease, chronic hepatitis, compensated liver cirrhosis, and decompensated liver cirrhosis patients were 0.831, $0.656,0.886$, and 0.984 , respectively.

Table 3. Diagnostic Accuracy of GDF-15 for Chronic Liver Disease

\begin{tabular}{lccccccc}
\hline & No. & AUROC & Cutoff value, ng/L & Se, \% & Sp, \% & PPV, \% & NPV, \% \\
\hline Chronic liver disease $^{*}$ & 145 & 0.831 & $>760$ & 69.0 & 92.1 & 92.6 & 67.5 \\
Chronic hepatitis & 54 & 0.656 & $>574$ & 53.7 & 79.2 & 58.0 & 76.2 \\
Compensated LC & 44 & 0.886 & $>760$ & 75.6 & 92.1 & 81.0 & 89.4 \\
Decompensated LC & 47 & 0.984 & $>869$ & 97.9 & 94.1 & 88.5 & 99.0 \\
\hline
\end{tabular}

GDF-15, growth differentiation factor 15; AUROC, area under the receiver operating characteristic curve; Se, sensitivity; Sp, specificity; PPV, positive predictive value; NPV, negative predictive value; LC, liver cirrhosis. *Included chronic hepatitis, compensated LC, and decompensated LC.

rate in endometrial cancers ${ }^{5}$ and increased incidence of oral leukoplakia, squamous cell carcinoma, and prostate cancer. ${ }^{6,7}$ Additionally, it was reported as the predicting factor of reoccurrence of colorectal cancer ${ }^{8}$ and the independent predicting factor of the mortality from cardiovascular diseases. ${ }^{9,10}$ In addition,
GDF-15 was reported to be related to abdominal obesity and insulin resistance, and it was reported as the predicting factors of insulin resistance. ${ }^{11}$

There are few papers on plasma levels of GDF-15 in chronic liver disease, but Liu et al. ${ }^{12}$ reported that GDF-15 increased the 
sensitivity and specificity of diagnosis of hepatocellular carcinoma accompanied with cirrhosis when measured with AFP. In addition to this paper, Si et al. ${ }^{13}$ reported that GDF-15 was associated with the pathogenesis of hepatitis $\mathrm{C}$ virus.

But so far, no studies to date have investigated the blood GDF-15 concentration in chronic liver diseases. This study is the first to confirm that patients with more severe chronic liver diseases had proportionately higher GDF-15 values.

Second, the increase in GDF-15 seemed to be caused by fibrosis rather than the hepatocellular damage by the infections in chronic liver diseases. To prove this, patients who had cancer, cancer recurrence, or noticeable increases in AST and ALT were excluded from this study to eliminate confounding factors that could arise in cases of cancer or acute infection. Moreover, there was no difference in the average AST and ALT values among groups of patients with chronic hepatitis, compensated liver cirrhosis, and decompensated liver despite clear differences in GDF-15 as well as strong correlations between the liver fibrosis and severity prediction models including CDS, CTP score, and MELD score $(r=0.447, r=0.474$, and $r=0.415$, respectively). In conclusion, increases in blood GDF-15 concentration were not due to hepatocellular damage from infection but appeared to be related to fibrosis level.

The relationship between GDF-15 and fibrosis of various tissues and organs is increasingly being described. Overexpression of GDF-15 caused by chronic and repetitive injuries of tissues or dysfunction of regulation the expression of this cytokine is a major factor in the pathogenesis of organ fibrosis. ${ }^{14-16}$

An increased GDF-15 expression was observed among others in patients with pulmonary, kidney, and liver fibrosis. ${ }^{17}$

In patients with chronic liver disease, the prolonged stimulation of hepatic stellate cells being the result of chronic damage to hepatocytes results in the release of profibrogenic abundant factors as GDF-15 and leads to the development of liver cirrhosis.

GDF-15 leads to the extracellular matrix (ECM) accumulation in the mechanism: (1) directly increasing the synthesis of ECM components as procollagen 1a, (2) inhibition of tissue collagenases expression, (3) increasing synthesis of ECM-degrading enzyme inhibitors (as plasminogen activator inhibitor type 1, tissue inhibitors of metalloproteinases). ${ }^{18-20}$

Patients with chronic liver diseases would have deposited not only extracellular protein but collagen when liver fibrosis occurred; continuous deposits of such substances in the liver would result in structural changes of the liver tissues and induce functional liver impairment, which ultimately leads to portal hypertension and clinical complications. Therefore, understanding liver fibrosis severity is important in predicting the prognosis of patients with chronic liver diseases and choosing treatment and surveillance periods; it is also used to assess treatment reactions.

Regarding studies of the relationships between GDF-15 and fibrosis, when Lambrecht et al. ${ }^{21}$ measured the serum GDF-15 concentration in 119 patients with systemic sclerosis, disease activity and organ involvement showed a strong relationship; in particular, more severe fibrosis in the liver showed a higher GDF-15 concentration. Lok et al. ${ }^{22}$ identified a relationship between myocardial fibrosis and GDF-15 in nonischemic dilated cardiomyopathy. No studies have been performed on the correlation between GDF-15 and liver fibrosis in patients with chronic hepatitis or liver cirrhosis. This study confirmed that there was a strong relationship between them.

Finally, the most critical result of this study was that GDF-15 showed extremely high sensitivity and specificity in distinguishing between chronic hepatitis and liver cirrhosis, and especially in distinguishing the patients with compensated and decompensated liver cirrhosis (at a cutoff of > $869 \mathrm{ng} / \mathrm{L}$; sensitivity, 97.9\%; specificity, 94.1\%).

Of the previous prediction models, CDS is a prediction model for liver fibrosis that is composed of platelets, AST/ALT ratio, and PT INR. A score $>8$ may reduce the need for liver biopsy for the diagnosis of liver fibrosis, ${ }^{23}$ but it fails to predict chronic liver disease severity. Also, CTP and MELD scores are applied to the prediction models for liver disease severity. CTP scoring had the following weaknesses: two of its five factors, ascites and hepatic coma, are not objective; all factors were treated the same regardless of weighted values, the cutoff value was arbitrarily set without any statistical basis; and patients within the same class were not uniform, so they could have different prognoses. $^{24}$

Unlike CTP score, MELD score is composed only of objective factors and gives weighted values to factors according to its importance. It is also continuous without the use of arbitrary restrictions. The strength of the MELD score was that even an increase in a single score was proportional to the risk, but it was ambiguous for selecting among the different factors used in multivariable analysis for completing the model and it ignored the importance of ascites; unlike CTP score, which could easily be calculated and applied to clinical settings, its calculation was complicated. ${ }^{24}$ Furthermore, most critically, it could not distinguish between or describe compensated and decompensated liver cirrhosis. ${ }^{1}$ In such cases, the use of GDF-15 is convenient for comparing CDS, CTP score, and MELD score and able to more accurately predict liver fibrosis and liver disease severity. In particular, GDF-15 is believed to be a biomarker that is capable of diagnosing compensated and decompensated liver cirrhosis, which is clinically critical.

Limitations of this study were expressed as follows. Firstly, because a small number of patient group, it was not possible to analyze association between GDF-15 according to the cause of chronic liver disease. And secondly, liver biopsy was not performed to examine the association of GDF-15 and fibrosis.

Despite having a number of limitations, our work generates important findings. In other words, this is the first study to investigate the effectiveness of GDP-15 in patients with chronic 
liver diseases, and our findings confirmed it to be a very valuable biomarker for predicting liver fibrosis and chronic liver disease severity. In particular, in the diagnosis of clinically important decompensated cirrhosis, it showed high diagnostic rate; when the cutoff valve of $869 \mathrm{ng} / \mathrm{L}$ was set, it had a sensitivity of $97.9 \%$ and specificity of $94.1 \%$.

\section{CONFLICTS OF INTEREST}

No potential conflict of interest relevant to this article was reported.

\section{REFERENCES}

1. D’Amico G, Garcia-Tsao G, Abraldes JG, et al. Prognostic indicators in cirrhosis. In: De Franchis R, ed. Portal hypertension IV: proceedings of the fourth Baveno International Consensus Workshop. Malden: Blackwell Publishing, 2005:53-103.

2. Mimeault M, Batra SK. Divergent molecular mechanisms underlying the pleiotropic functions of macrophage inhibitory cytokine-1 in cancer. J Cell Physiol 2010;224:626-635.

3. de Jager SC, Bermúdez B, Bot I, et al. Growth differentiation factor 15 deficiency protects against atherosclerosis by attenuating CCR2mediated macrophage chemotaxis. J Exp Med 2011;208:217-225.

4. Ginés P, Quintero E, Arroyo V, et al. Compensated cirrhosis: natural history and prognostic factors. Hepatology 1987;7:122-128.

5. Staff AC, Trovik J, Eriksson AG, et al. Elevated plasma growth differentiation factor-15 correlates with lymph node metastases and poor survival in endometrial cancer. Clin Cancer Res 2011;17:48254833.

6. Yang CZ, Ma J, Luo QQ, et al. Elevated level of serum growth differentiation factor 15 is associated with oral leukoplakia and oral squamous cell carcinoma. J Oral Pathol Med 2014;43:28-34.

7. Tsui KH, Chang YL, Feng TH, et al. Growth differentiation factor-15 upregulates interleukin-6 to promote tumorigenesis of prostate carcinoma PC-3 cells. J Mol Endocrinol 2012;49:153-163.

8. Wallin U, Glimelius B, Jirström K, et al. Growth differentiation factor 15: a prognostic marker for recurrence in colorectal cancer. Br J Cancer 2011;104:1619-1627.

9. Wollert KC, Kempf T, Peter T, et al. Prognostic value of growthdifferentiation factor-15 in patients with non-ST-elevation acute coronary syndrome. Circulation 2007;115:962-971.

10. Khan SQ, Ng K, Dhillon 0, et al. Growth differentiation factor-15 as a prognostic marker in patients with acute myocardial infarction. Eur Heart J 2009;30:1057-1065.

11. Kempf T, Guba-Quint A, Torgerson J, et al. Growth differentiation factor 15 predicts future insulin resistance and impaired glucose control in obese nondiabetic individuals: results from the XENDOS trial. Eur J Endocrinol 2012;167:671-678.

12. Liu X, Chi X, Gong Q, et al. Association of serum level of growth differentiation factor 15 with liver cirrhosis and hepatocellular carcinoma. PLoS One 2015;10:e0127518.

13. Si Y, Liu X, Cheng M, et al. Growth differentiation factor 15 is induced by hepatitis $\mathrm{C}$ virus infection and regulates hepatocellular carcinoma-related genes. PLoS One 2011;6:e19967.

14. Krzemien S, Knapczyk P. Current review on the role of transforming growth factor beta (TGF-beta) in some pathological disorders. Wiad Lek 2005;58:536-539.

15. Kajdaniuk D, Marek B, Borgiel-Marek H, Kos-Kudła B. Vascular endothelial growth factor (VEGF)-part 1: in physiology and pathophysiology. Endokrynol Pol 2011;62:444-455.

16. Kajdaniuk D, Marek B, Foltyn W, Kos-Kudła B. Vascular endothelial growth factor (VEGF)-part 2: in endocrinology and oncology. Endokrynol Pol 2011;62:456-464.

17. Blobe GC, Schiemann WP, Lodish HF. Role of transforming growth factor beta in human disease. N Engl J Med 2000;342:1350-1358.

18. Matsuzaki K, Murata M, Yoshida K, et al. Chronic inflammation associated with hepatitis $\mathrm{C}$ virus infection perturbs hepatic transforming growth factor beta signaling, promoting cirrhosis and hepatocellular carcinoma. Hepatology 2007;46:48-57.

19. Tahashi Y, Matsuzaki K, Date M, et al. Differential regulation of TGF-beta signal in hepatic stellate cells between acute and chronic rat liver injury. Hepatology 2002;35:49-61.

20. Shah R, Reyes-Gordillo K, Arellanes-Robledo J, et al. TGF-beta1 up-regulates the expression of PDGF-beta receptor mRNA and induces a delayed PI3K-, AKT-, and p70(S6K)-dependent proliferative response in activated hepatic stellate cells. Alcohol Clin Exp Res 2013;37:1838-1848.

21. Lambrecht S, Smith V, De Wilde K, et al. Growth differentiation factor 15, a marker of lung involvement in systemic sclerosis, is involved in fibrosis development but is not indispensable for fibrosis development. Arthritis Rheumatol 2014;66:418-427.

22. Lok SI, Winkens B, Goldschmeding R, et al. Circulating growth differentiation factor-15 correlates with myocardial fibrosis in patients with non-ischaemic dilated cardiomyopathy and decreases rapidly after left ventricular assist device support. Eur J Heart Fail 2012;14:1249-1256.

23. Bonacini M, Hadi G, Govindarajan S, Lindsay KL. Utility of a discriminant score for diagnosing advanced fibrosis or cirrhosis in patients with chronic hepatitis C virus infection. Am J Gastroenterol 1997;92:1302-1304.

24. O'Leary JG, Friedman LS. Predicting surgical risk in patients with cirrhosis: from art to science. Gastroenterology 2007;132:16091611. 